\title{
Adult intussusception: An unlikely diagnosis
}

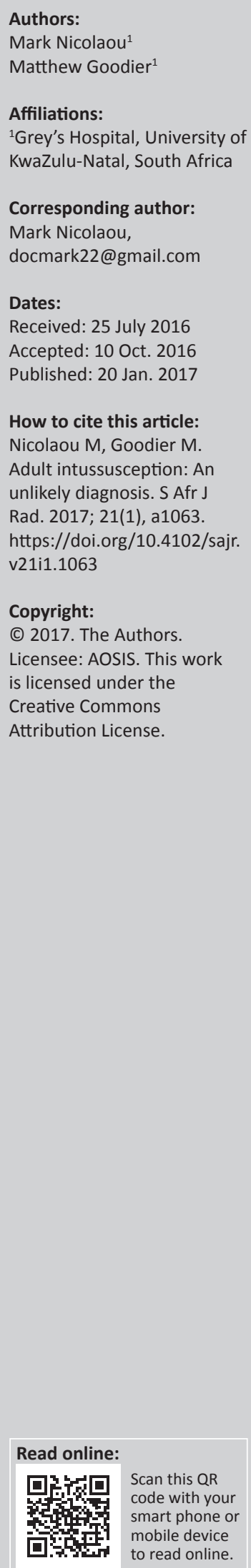

Intussusception happens when a portion of bowel telescopes into the adjacent bowel segment. It is a rare condition in the adult population, particularly in the young adult, and poses a diagnostic dilemma to the attending clinician because of its variable clinical presentation. Radiological imaging is important in the early diagnosis, and it allows prompt surgical management to avoid severe complications, particularly bowel ischaemia and necrosis. This case report describes the clinical and multimodality radiological findings of an ileocolic intussusception secondary to a mucosa-associated lymphoid tissue (MALT) lymphoma in a young adult patient.

\section{Introduction}

Intussusception happens when a portion of the digestive tract becomes telescoped (intussusceptum) into the adjacent bowel segment (intussuscipiens) as a result of peristalsis. ${ }^{1,2}$ Primary or idiopathic causes are unknown and occur in the small intestine. Secondary cause is thought to initiate from a mural bowel lesion or irritant that serves as a lead point and causes altered peristalsis. Intussusception is uncommon in adults. It is estimated that only $5 \%$ of all intussusceptions occur in adults, and only $1 \%-5 \%$ of bowel obstructions in adults are the result of an intussusception. ${ }^{3}$ The majority of cases in adults are because of the secondary causes with an identifiable lead point discovered intra-operatively. ${ }^{1,3}$ Conversely, paediatric causes are usually primary and can be managed non-operatively with pneumatic enema in $80 \%$ of patients. ${ }^{1}$

\section{Case presentation}

A 24-year-old male patient known with retroviral disease and previously treated pulmonary tuberculosis (TB) presented with a 1-day history of sudden right iliac fossa pain associated with emesis and obstipation.

On arrival, an ultrasound of the abdomen was performed, which showed several loops of dilated, fluid-filled bowel in the right side of the abdomen as well as a bowel-within-bowel appearance with a concentric wall thickening ('doughnut sign') which was concerning for an ileocolic intussusception as shown in Figure 1.

A computed tomography (CT) of the abdomen (Figure 2) was performed which confirmed invagination of the terminal ileum into the ascending colon. A rounded enhancing mass adjacent to the medial aspect of the intussusceptum was thought to be the lead point of the intussusception. There was evidence of high-grade small bowel obstruction and possible ischaemic necrosis of the involved bowel.

The patient proceeded to an emergency laparotomy, and a bowel resection involving the terminal ileum, caecum and proximal ascending colon was performed. Histopathological examination of the resected specimen demonstrated a mucosal and submucosal tumour, as the lead point, which had typical features of a low-grade B-cell mucosa-associated lymphoid tissue (MALT) lymphoma. The patient had an uneventful recovery period post-operatively.

\section{Discussion}

The clinical presentation of adult intussusception is variable and symptoms are often nonspecific. The majority of cases in adults present with features of a partial bowel obstruction such as nausea, vomiting and constipation. Melena and an abdominal mass may also be present. ${ }^{3}$ Classification of intussusception is either anatomical (e.g. ileocolic or enteroenteric) or may be pathological, based on the underlying pathology. ${ }^{2}$ In the adult population, common underlying lead points in the small intestine can be due to the presence of intra- or extraluminal lesions such as a Meckel's diverticulum, inflammatory bowel lesions, postoperative adhesions, lipoma, 


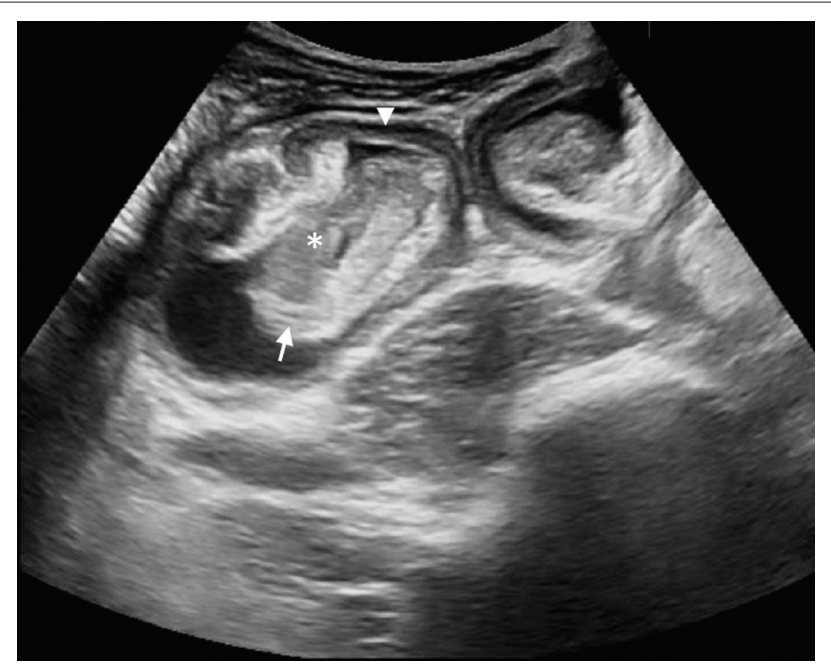

Source: Ultrasound Department, Grey's Hospital Radiology, Pietermaritzburg (obtained from actual patient)

FIGURE 1: Axial ultrasound of the abdomen depicting a bowel-within-bowe appearance with concentric thickening of the bowel (arrowhead), with the mesentery of the intussusceptum (arrow) dragged into the intussuscipiens (asterisk).

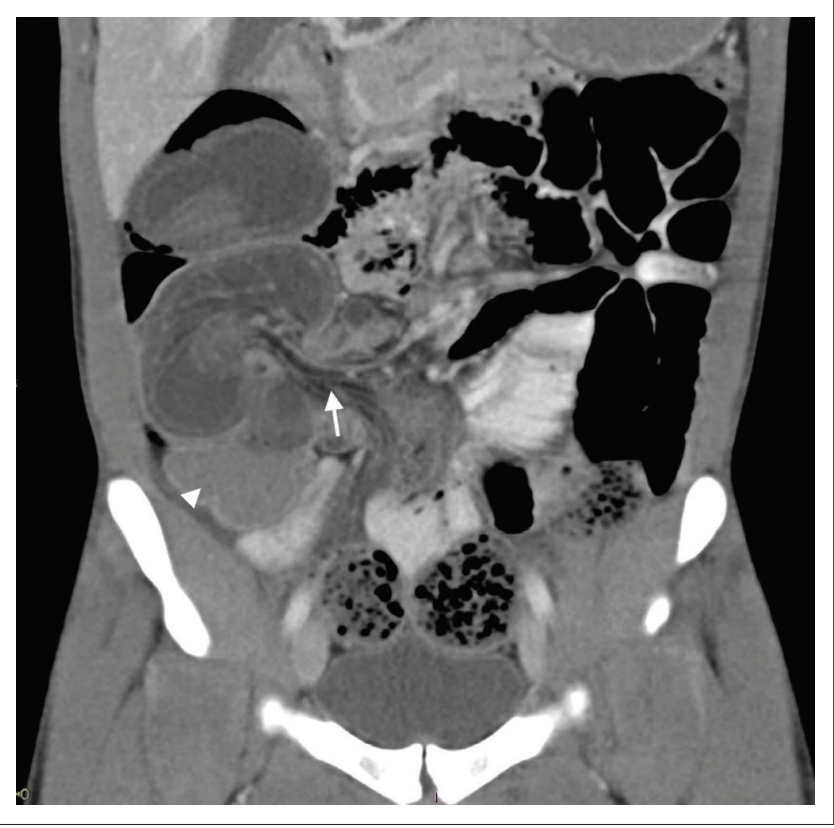

Source: CT Department, Grey's Hospital Radiology, Pietermaritzburg (obtained from actual patient)

FIGURE 2: Contrast-enhanced coronal computed tomography of the abdomen demonstrating invagination of the terminal ileum along with its mesentery (arrow) into the ascending colon (arrowhead). Histopathological examination of the resection specimen found the lead point to be a submucosal tumour with features of a low-grade B-cell MALT Iymphoma.

adenomatous polyps, lymphoma and metastases. Iatrogenic causes have been described whereby indwelling intestinal tubes can act as a lead point. Adenocarcinoma is seen in approximately one-third of cases involving the small intestine. Malignant adenocarcinoma forms the majority of causes of intussusception in the large bowel..$^{1,3}$ Typically, a mucosal lesion serves as a lead point, whereby peristalsis at this point causes invagination of the proximal bowel segment into the distal bowel segment. ${ }^{3}$ This leads to further obstruction of intestinal contents causing more vigorous peristalsis in the affected segment, which causes further telescoping of the intussuscepted segment. Eventually, blood flow within the mesentery of the intussusceptum is compromised, with oedema leading to further obstruction and ultimately bowel ischaemia. ${ }^{3,4}$ Although a lead point is usually present in adult intussusception, $20 \%$ of cases are idiopathic.

In our particular case, it is peculiar that a non-benign lesion was the lead point, given his relatively young age. However, it has become apparent in recent literature that an increased incidence of intussusception has been reported in patients with AIDS. This is understood to be due to the high incidence of infectious and neoplastic conditions of the bowel in patients with AIDS, such as lymphoid hyperplasia, Kaposi's sarcoma and non-Hodgkin's lymphoma. ${ }^{5}$ Of particular interest is that the average age for intussusception in an HIVpositive adult was lower (41 years) compared with 63 years in a non-HIV-infected patient in a study performed by Wood et al. ${ }^{6}$ This is important for clinicians to be aware of in regions where HIV is prevalent.

Abdominal radiographs are typically non-specific but may demonstrate the presence and level of bowel obstruction. Ultrasound is the most useful initial imaging modality. Classical imaging findings include the 'target' or 'doughnut' signs in the transverse view and the 'pseudokidney' sign or 'hayfork' sign in the longitudinal view. ${ }^{1,7}$ An abdominal CT is currently considered as the most sensitive radiological method to confirm intussusception. ${ }^{3}$ The characteristic features of CT scan include an inhomogeneous target sign consisting of the layers of bowel or a sausage-shaped soft tissue mass with a layered effect; in addition, mesenteric fat and vessels within the bowel lumen may also be seen on contrast-enhanced scans. In the setting of a suspected intussusception, CT can provide valuable additional information, including not only confirming the diagnosis but also delineating the location, the nature of any associated masses and its relationship of the affected bowel to surrounding tissues. It can also aid in staging a patient with a suspected nonbenign cause for intussusception. Abdominal CT may be able to differentiate between intussusception without a lead point from those with a lead point, which is key in planning subsequent management. ${ }^{2,3,4}$ Management in the adult population is surgical with primary resection of the involved bowel with either end-to-end anastomosis of the resected ends in a non-emergent setting or a defunctioning colostomy with repair at a later stage., ${ }^{2,4}$

\section{Conclusion}

Intussusception in the adult population poses a diagnostic dilemma for the attending clinician due to its rarity and vague clinical presentation. Ultrasound is often the most useful initial investigation with CT providing useful adjunctive information in many cases allowing the management team to establish not only the cause of the bowel obstruction but also its location and the presence and nature of the lead point. 


\section{Acknowledgements}

The author thanks Dr Matthew Goodier, the head clinical unit consultant radiologist, Grey's Hospital, Pietermaritzburg, South Africa.

\section{Competing interests}

The authors declare that they have no financial or personal relationships which may have inappropriately influenced them in writing this article.

\section{Authors' contributions}

M.N. followed up the patient and wrote the case report. M.G. proof read the case report and assisted with image capture and editing.

\section{References}

1. Marinis A, Yiallourou A, Samanides L, et al. Intussusception of the bowel in adults: A review. World J Gastroenterol. 2009;15(4):407-411. http://dx.doi.org/10.3748/ wjg.15.407

2. Kim $Y H$, Blake $M A$, Harisinghani $M G$, et al. Adult intestinal intussusception: $C T$ appearances and identification of a causative lead point. Radiographics 2006; 26:733-744. http://dx.doi.org/10.1148/rg.263055100

3. Azar T, Berger DL. Adult intussusception. Ann Surg. 1997;226:134-138. http:// dx.doi.org/10.1097/00000658-199708000-00003

4. Begos DG, Sandor A, Modlin IM. The diagnosis and management of adult intussusception. Am J Surg 1997;173:88-94. http://dx.doi.org/10.1016/S0002 9610(96)00419-9

5. Gayer G, Zissin R, Apter S, Papa M, Hertz M. Pictorial review: Adult intussusceptionA CT diagnosis. Br J Radiol. 2002;75(890):185. http://dx.doi.org/10.1259/bjr.75. 890.750185

6. Wood BJ, Kumar PN, Cooper C, Silverman PM, Zeman RK. AIDS-associated intussusception in young adults. J Clin Gastroenterol. 1995;21(2):158-162. http:// dx.doi.org/10.1097/00004836-199509000-00019

7. Weissberg DL, Scheible W, Leopold GR. Ultrasonographic appearance of adult intussusception. Radiology. 1977;124:791-792. http://dx.doi.org/10.1148/ 124.3.791 